

\title{
RESUMEN
}

Este artículo explora la experiencia vivida de los trabajadores de residuos electrónicos (ewaste) en Agbogbloshie, un centro primario de reciclaje de desechos electrónicos y un mercado de chatarra en Accra, Ghana, que ha sido descrito como uno de los lugares más contaminados de la Tierra. Para formular una comprensión más crítica y contextual de las vidas de estos trabajadores y su experiencia corporal tóxica, el artículo presenta el concepto de «corporalidad poscolonial tóxica», un término que se despliega para navegar y afrontar la controvertida intersección entre cuerpos, toxinas e intervenciones de reciclaje «verde» en un desguace urbano poscolonial. Después de presentar las dimensiones sociales, económicas y tóxicas de Agbogbloshie, este artículo trata en general sobre los problemas y las políticas de la permeabilidad tóxica, y en particular sobre las fronteras precarias de los cuerpos, ambientes y prácticas de mitigación de riesgos en un contexto poscolonial. Se argumenta que la corporalidad poscolonial tóxica proporciona una perspectiva crítica de la salud ambiental, que puede informar a los compromisos antropológicos con las vidas que navegan por los mundos permeables y entrecruzados de la contaminación y la poscolonización.

PALABRAS CLAVE

Tóxicos, residuos electrónicos, embodiment, poscolonialismo, Ghana.

\section{BODIES, TOXINS, AND E-WASTE LABOUR INTERVENTIONS IN GHANA: TOWARD A TOXIC POSTCOLONIAL CORPORALITY?}

\begin{abstract}
This article explores the lived experience of electronic waste (e-waste) workers in Agbogbloshie, a primary e-waste recycling center and scrap metal market in Accra, Ghana, that has been described by international environmental organizations as one of the most polluted places on Earth. To formulate a more critical and contextual understanding of the lives of these e-waste workers and their embodied toxic experience, the article introduces the concept of "toxic postcolonial corporality", a term deployed to navigate and confront the contentious intersection of bodies, toxins, and "green" recycling interventions in a postcolonial urban scrapyard. After introducing the social, economic, and toxic dimensions of Agbogbloshie, I discuss the problems and politics of permeability in general and the precarious boundaries of bodies, environments, and risk mitigation practices in a postcolonial context in particular. It will be argued that the notion of toxic postcolonial corporality offers a possible critical environmental health perspective that can inform anthropological engagements with the embedded lives navigating the permeable and intersecting worlds of pollution, postcolonization, and decontamination.
\end{abstract}

KEY WORDS

Toxics, e-waste labor, embodiment, postcolonialism, Ghana. 


\section{Introducción}

Agbogbloshie, un bullicioso desguace de chatarra en Accra, Ghana, se ha convertido en un lugar significativo para académicos y activistas que realizan estudios globales sobre residuos electrónicos (e-waste) y toxicidad. En tanto que vibrante emplazamiento de políticas simbólicas y materiales en torno a los despojos de la era digital y los desastres medioambientales, Agbogbloshie ha atraído a organizaciones internacionales de defensa ambiental, ingenieros, científicos de salud ambiental, turistas de los suburbios y lo tóxico, periodistas, fotógrafos y científicos sociales. Con el apoyo de una serie de agencias gubernamentales y organizaciones no gubernamentales (ONG), Agbogbloshie también se ha transformado en un espacio experimental para intervenciones de salud ambiental, y actualmente alberga una nueva instalación de reciclaje de residuos electrónicos destinada a cumplir los objetivos de reducción de riesgos tóxicos. Estas intervenciones tienen como propósito principal reducir la exposición tóxica y los riesgos para la salud asociados a la «quema de cables» —una fuente primaria de contaminación del aire en Agbogbloshie-, al alentar el uso de máquinas automáticas para pelar cables con aislamiento de varios tamaños, que contienen cobre y otros materiales que son valiosos y, sin embargo, tóxicos. Los trabajadores de Agbogbloshie ocupan una zona humeante del desguace de chatarra donde se da una intensa actividad de recuperación de metales, un lugar donde la quema de residuos electrónicos para recuperar metales valiosos, especialmente cobre y aluminio, es una actividad cotidiana de "minería urbana» (Grant y Oteng-Ababio, 2016).

Inundado por maravillas «modernas» desechadas, desde coches abandonados hasta frigoríficos, cintas de correr, fotocopiadoras y voluminosos ordenadores de sobremesa, Agbogbloshie es también un espacio urbano consumido por el plomo, el mercurio, el cadmio, los PCB y varios contaminantes del aire, incluyendo éteres difenílicos polibromados (PBDE). Estas toxinas han sido el foco de numerosos estudios de salud ambiental en Agbogbloshie y en las áreas circundantes (Caravanos, Clark, Fuller y Lambertson, 2011; Caravanos, Clarke y Osei, 2013; Feldt, Fobil y Wittsiepe, 2014; Wittsiepe, Fobil y Till, 2015). Para los recicladores de residuos electrónicos, que trabajan con cables eléctricos con base de cobre, el problema principal de exposición es la quema del plástico de policloruro de vinilo (PVC), que aísla los cables y contiene compuestos nocivos. Aquellos que viven y trabajan en centros de reciclaje de residuos electrónicos han sido el foco de 
numerosos estudios epidemiológicos ambientales (Asante, Adu-Kumi y Nakahiro, 2011; Asante, Agusa y Biney, 2012; Caravanos, Clarke y Osei, 2013; Feldt, Fobil y Wittsiepe, 2014; Liu, Xu y Wu, 2011; Sepúlveda, Schluep y Renaud, 2010; Wittsiepe, Fobil y Till, 2015). Estos estudios han demostrado que los niños son particularmente vulnerables a causa del comportamiento mano-a-boca común entre los niños pequeños, y también por otras vías de exposición, que incluyen la lactancia materna, la exposición placentaria, la exposición a la ropa contaminada de los padres y el contacto piel-a-piel. La investigación también ha revelado que los niños que viven en las instalaciones de reciclaje de residuos electrónicos, o cerca de estas, muestran pesos, alturas e índices de masa corporal significativamente bajos en comparación con poblaciones no afectadas por los contaminantes del reciclaje informal de residuos electrónicos. El plomo, presente en muchos de estos lugares, también afecta al desarrollo neurológico neonatal (Liu, Xu y Wu, 2011). Estos hallazgos han llevado a varios investigadores a alentar a las agencias internacionales de salud pública a admitir que "los efectos en la salud por la exposición a los residuos electrónicos deben convertirse en una prioridad de la comunidad internacional» (Grant, Goldizen y Sly, 2013: 357).

Estos peligros tóxicos, bien conocidos, han atraído la atención de ONG internacionales, convirtiendo Agbogbloshie en un lugar de intervenciones optimistas, aunque controvertidas, que buscan "eliminar» la quema de productos electrónicos por parte de los trabajadores migrantes que conforman la mayoría de los trabajadores de los desguaces. Estas intervenciones «verdes» incluyen lógicas y objetivos de salud ambiental que tienden a pasar por alto los cuerpos permeables y las subjetividades que habitan en este paisaje poscolonial tóxico. En este artículo, me baso en investigación etnográfica para explorar cómo los trabajadores de Agbogbloshie narran, entienden y se refieren a su propio sufrimiento corporal para dar sentido a los riesgos ambientales y de salud ocupacional a los que se enfrentan. En particular, me centro en cómo los trabajadores relatan no solamente padecimientos relacionados con toxicidad "interna" (por ejemplo, dolores en los pulmones y el corazón), sino también condiciones «exteriores" más visibles de sus cuerpos — a menudo la piel quemadapara dar sentido a su propia corporalidad tóxica. Además de explorar cómo la corporalidad y la experiencia tóxica rompen o reconfiguran las demarcaciones entre cuerpo y medio ambiente, también pongo de manifiesto las formas en que la toxicidad y la corporalidad se convierten en el lugar central de loables esfuerzos de mitigación de ries- 
gos para la salud ambiental, que irónicamente no logran transformar o reducir la corporalidad tóxica en un contexto poscolonial duradero. De esta manera, exploro cómo una intervención basada en soluciones pasa por alto la complejidad y la diversidad de las relaciones eco-corporales en un desguace donde se cruzan cuerpos, toxinas y necesidades económicas.

\section{Breve descripción de Agbogbloshie}

Agbogbloshie ha sido descrito como uno de los barrios marginales más tóxicos del mundo y ha devenido un hito para los dispositivos electrónicos desechados del mundo, convirtiéndose en una de las zonas de residuos más grandes del mundo en general y de África Occidental en particular. La Asamblea Metropolitana de Accra (AMA) ${ }^{1}$ es consciente de los peligros que representan las actividades informales de reciclaje de residuos electrónicos, no solo para el medio ambiente, sino también para las personas que viven y trabajan en Agbogbloshie y sus alrededores. Sin embargo, la AMA y otras agencias gubernamentales se han mostrado reticentes a remediar el problema a causa del capital que generan estas actividades. Los académicos que han revisado las evaluaciones políticas y económicas de Agbogbloshie indican que el mercado de residuos electrónicos genera entre 105 y 268 millones de dólares anuales y brinda oportunidades de empleo para aproximadamente 4.500-6.000 trabajadores (Daum, Stoler y Grant, 2017). Después de una devastadora inundación en 2015, que mató a varios centenares de personas, la AMA recurrió a acciones agresivas de mitigación de inundaciones que afectaron directamente a los trabajadores que viven al lado de Agbogbloshie, en el asentamiento informal Old Fadama, un asentamiento que alberga a la mayoría de los trabajadores de Agbogbloshie. La AMA demolió las viviendas de la zona de inundación, desplazando a muchas personas y causando indignación y resistencia entre los residentes de Old Fadama.

1. El objetivo de la AMA es «mejorar la calidad de vida de la gente de la ciudad de Accra, especialmente los pobres, vulnerables y excluidos, proporcionando y manteniendo servicios básicos e instalaciones en las áreas de educación, salud, saneamiento y otros servicios sociales, en un contexto de disciplina, sentido de la urgencia y compromiso con la excelencia». 


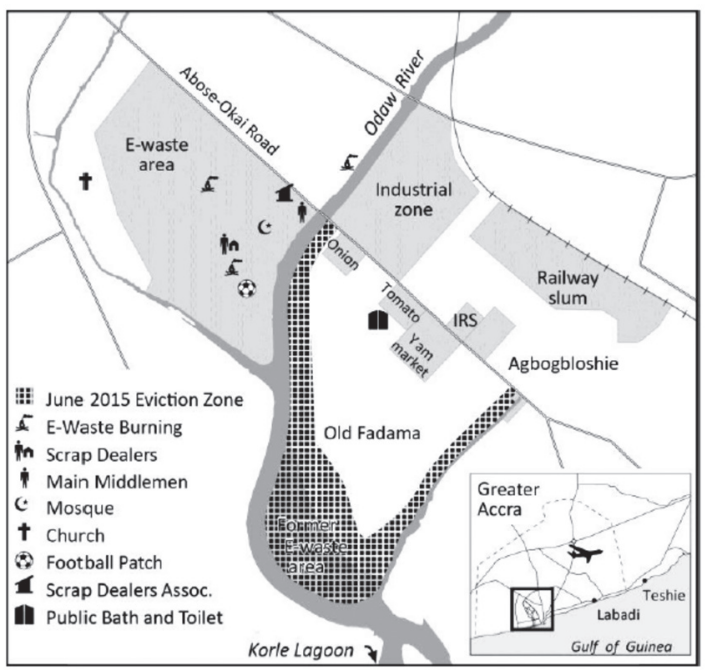

FIGURA 1. Plano de Agbogbloshie. Adaptado de Daum, Stoler y Grant (2017).

Agbogbloshie es más que un desguace de chatarra. A lo largo de los años, el lugar ha crecido hasta incluir mezquitas, iglesias, campos de fútbol informales, un pasto para cabras y jardines con productos agrícolas (ver Figura 1). Con un mercado de carne a poca distancia a pie del desguace, el ganado pasta por todas partes e incluso descansa en los montículos de ceniza generados por la quema de cables. Durante una visita en 2016, un trabajador me explicó que "[el ganado] siempre está aquí. Se sientan en la ceniza. Después de que quemamos, esperan y se sientan en la ceniza». Observé esto durante mis visitas diarias al lugar y luego me dijeron que, cuando la ceniza se enfría, al ganado le gusta descansar y restregársela por el pelo. «Les gusta la sensación», me dijeron. Estudios recientes realizados por investigadores de la Universidad de Ghana han confirmado que la carne de este ganado es rica en metales pesados y otras sustancias tóxicas, y que los mismos contaminantes se encuentran en la sangre de los trabajadores, especialmente niveles altos de metales pesados (por ejemplo, plomo, zinc y cromo) y productos retardantes de llama. La poca o ninguna protección que llevan estos trabajadores ha sido entendida como la causa de estos niveles elevados de toxinas, pero los efectos a largo plazo para la salud que estas prácticas tienen sobre los trabajadores aún no se han descubierto (ver Figura 2). 


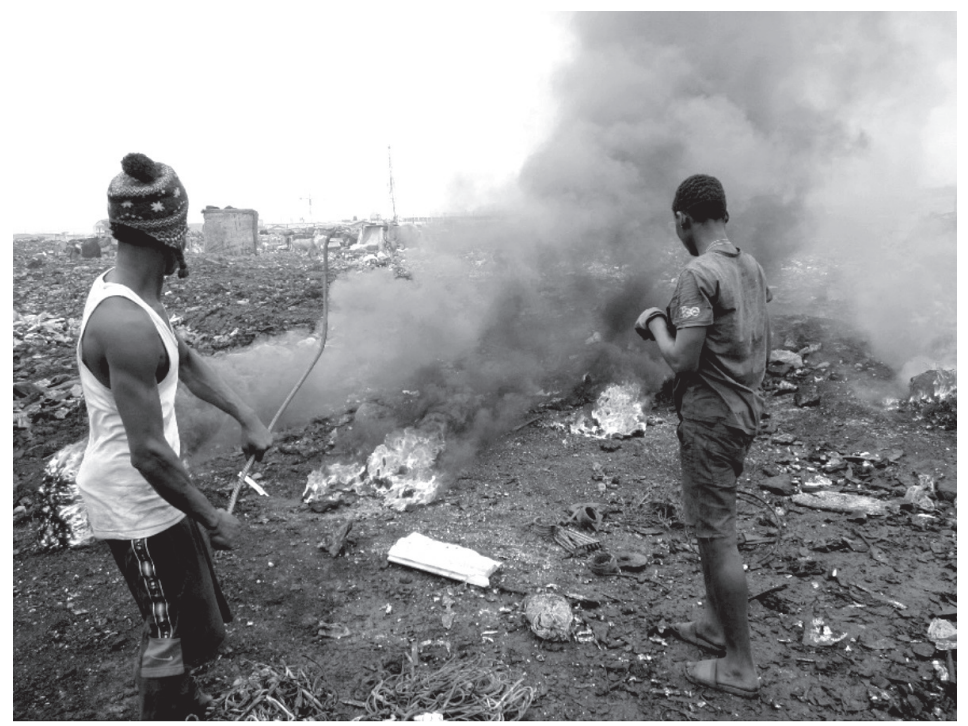

Figura 2. Quema de residuos electrónicos en Agbogbloshie. Fotografía del autor.

El reciclaje informal de residuos electrónicos en Agbogbloshie ha causado, sin duda, efectos medioambientales perjudiciales. La quema a cielo abierto de residuos de aparatos eléctricos y electrónicos (RAEE) para extraer cobre y aluminio contamina directamente el aire, el agua y la tierra de los alrededores. Una vez que la chatarra y los RAEE se transportan a Agbogbloshie, ya sea desde hogares y negocios cercanos o desde el puerto de Tema, los trabajadores toman lo que saben que pueden desmantelar y revenden el metal por dinero. Además del desmantelamiento de ordenadores, impresoras y otros productos electrónicos, los trabajadores también realizan quemas al aire libre de RAEE para extraer cobre y aluminio. Esta ha sido la principal fuente de contaminación del aire y es el principal riesgo para la salud ambiental identificado por epidemiólogos reconocidos internacionalmente y expertos en salud pública del Servicio de Salud de Ghana, la principal agencia de salud pública del país. Es bien sabido que estas prácticas informales de reciclaje de residuos electrónicos liberan toxinas no solo en el aire, sino que también dejan una ceniza tóxica en el suelo, que luego puede volar y convertirse en otra toxina del aire, así como en una fuente de contaminación del agua local. Además, se culpa a la contaminación generada en Agbogbloshie por la mala calidad del aire en Accra (Daum, Stoler y Grant, 2017), aunque muchos saben que 
esta ciudad de más de dos millones de habitantes también está ahogada por automóviles, autobuses y camiones de reparto que contribuyen significativamente a la calidad del aire urbano.

Según un informe sobre las condiciones laborales del trabajo con residuos electrónicos:

\begin{abstract}
El reciclaje de residuos electrónicos en Ghana está organizado en muchas empresas pequeñas e informales. Los trabajadores del reciclaje en Accra provienen principalmente de la parte pobre del norte del país, una región que se enfrenta a una situación de inseguridad alimentaria crónica. El reciclaje de residuos electrónicos se ha revelado como una estrategia de subsistencia más fiable, a pesar de los graves peligros ambientales y para la salud. La mayoría de las personas empleadas en este sector tienen entre 14 y 40 años de edad, trabajan entre 10 y 12 horas por día y realizan entre 108 y 168 horas extra por mes. Sin embargo, la mayoría de los trabajadores no tienen horas de trabajo fijas por día o por semana (Lundgren, 2012: 28).
\end{abstract}

Mi propia investigación etnográfica en Agbogbloshie también reveló que la mayoría de estos trabajadores preferirían estar en sus aldeas en el norte, especialmente si su subsistencia pudiera ser mantenida por salarios iguales a los del sector del reciclaje de residuos electrónicos. Según Alhassan, por ejemplo, que ha trabajado en Agbogbloshie desde 2014, "La vida en el norte es dura. Sin dinero. No hay trabajo alli. Por eso venimos aquí. Aqui es difícil. Nadie se preocupa por nosotros. Vivir al norte con trabajo es bueno. Lo deseo". Como se describirá más adelante, poner sus cuerpos (y su salud) en la primera línea tóxica se considera la mejor de las opciones disponibles para los trabajadores que sienten y encarnan el dolor de la vulnerabilidad, el desplazamiento y el malestar causados por situaciones de extrema pobreza y marginación.

En este artículo exploro lo que he llamado «corporalidad poscolonial tóxica» para desarrollar una crítica social, ambiental y biopolítica de los límites inciertos entre el trabajo de descarte electrónico y las lógicas paradójicas de mitigación del riesgo en las intervenciones de las ONG. La forma en que los trabajadores de Agbogbloshie entienden y participan de un discurso de permeabilidad y precariedad arroja luz sobre las maneras de dar sentido a sus cuerpos, entornos y economías poscoloniales. Argumentaré que estos discursos plantean un nuevo punto de vista antropológico en la intersección de los estudios de los desechos (Discard Studies) y la «ecología política del cuerpo» (Alter, 2016) en un lugar de recuperación extrema y tóxica de metales. En particular, sostengo que prestar atención a la forma en que estos trabajadores gestionan y dan sentido a los riesgos ambientales para la salud del trabajo con residuos 
electrónicos (Chen, Dietrich y Huo, 2011; Feldt, Fobil y Wittsiepe, 2014) y cómo lidian con las incertidumbres vitales del trabajo y las intervenciones de las ONG, nos ayuda a comprender el malestar corporal y la ecología tóxica del reciclaje informal de residuos electrónicos, y especialmente las experiencias corporales de la poscolonialidad tóxica.

Diversas preguntas críticas sobre salud ambiental conforman el enfoque de este artículo y podrían utilizarse para potenciar teóricamente los debates sobre el trabajo tóxico y las relaciones cuerpo-ambiente: ¿a qué se enfrentan los cuerpos trabajadores y las subjetividades en un contexto urbano poscolonial tóxico? ¿Qué historias cuentan las subjetividades y los cuerpos que trabajan con desechos electrónicos? ¿Qué límites presionan o quiebran estas historias de corporalidades tóxicas? Finalmente, ¿cuál es el papel ético y político de la antropología en las luchas tóxicas poscoloniales, especialmente cuando persisten desafíos extremos de salud ambiental y cuando las intervenciones basadas en soluciones pasan por alto la complejidad y la diversidad de las relaciones eco-corporales? ¿De qué manera estas intervenciones reducen la comprensión del riesgo a la toxicidad química en lugar de tener en cuenta una ecología más amplia del riesgo que condiciona realmente las experiencias corporales del trabajo tóxico?

\section{Contextualización poscolonial}

Es difícil comprender totalmente la política de salud ambiental del trabajo y la vida con residuos electrónicos en Agbogbloshie sin una apreciación completa del contexto colonial, poscolonial y neocolonial de Ghana en general. Ghana se convirtió en un Estado-nación independiente en 1957, en una ruptura con los británicos liderada por Kwame Nkrumah, que provocó un movimiento de independencia panafricano. La desigualdad de la geografía económica mundial no cambió drásticamente como resultado de este movimiento de independencia. Una de las razones fue que muchas empresas británicas permanecieron allí después de 1957 y, de hecho, poseían el monopolio de la industria minera de Ghana. Actualmente, China es la nueva potencia extranjera que está expandiendo operaciones mineras y financiando proyectos de desarrollo en todo el país. África no es pobre, dice el dicho, sino que su riqueza la roban. Tal y como lo expresó el propio Nkrumah en 1965 en su libro Neo-Colonialism: The Last Stage of Imperialism, "África es una paradoja que ilustra y pone de relieve el neocolonialismo. Su tierra es rica, pero los productos que vienen de encima y debajo de esa tierra continúan enriqueciendo, no principal- 
mente a africanos, sino a grupos e individuos que operan para empobrecer a África».

Si bien estudios recientes sobre Agbogbloshie afirman que «los residuos electrónicos son un fenómeno multidimensional» que requiere una "trayectoria más sostenible para las políticas sobre residuos electrónicos" (Daum, Stoler y Grant, 2017), la crítica poscolonial y la poscolonialidad en sí carecen de implicación o alusión directa a este tema. Esta omisión puede que no sea intencional, pero enfrentarse a la poscolonialidad es fundamental si realmente deseamos desarrollar una comprensión más profunda de las políticas de salud ambiental y del contexto tóxico en Agbogbloshie. Además, es importante porque la poscolonialidad en Ghana está incesantemente condicionada por las actuales luchas bioculturales y ecosociales que dan forma a los procesos de descolonización. Como expresó Fanon en The Wretched of the Earth, la vida bajo la descolonización tiene que ver con delincuencias duraderas y formas de lucha semiótica que rara vez, si alguna, escapan a la ruptura violenta: "Liberación nacional, renacimiento nacional, restauración de la nación para la gente, o para la Commonwealth, cualquiera que sea el nombre que se use, cualquiera que sea la última expresión, la descolonización siempre es un suceso violento" (Fanon, 1963: 1). Ese "suceso», tal y como han sostenido durante mucho tiempo las ciencias sociales poscoloniales y fenomenológicas, está corporalmente materializado y, por lo tanto, se desarrolla en cuerpos reales que están unidos a lugares y que están condicionados por historias de lucha. Dar sentido al cuerpo poscolonial contaminado en Agbogbloshie es una cuestión de considerar la toxicidad en medio de formas significativas de violencia e injusticia corporales y ambientales (Akese y Little, 2018; Peluso y Watts, 2001). En otras palabras, la lucha por la salud ambiental y ante la injusticia tóxica en Agbogbloshie es una lucha a la que da forma una historia más profunda de explotación y marginación que persiste en la Ghana poscolonial. Según Pierre (2013: XII): "Como poscolonia, las realidades contemporáneas en Ghana están relacionadas con un conjunto interconectado de prácticas, experiencias y sistemas de creencias, una disyuntiva especifica en la larga historia de la construcción del imperio europeo». En medio de esta construcción imperialista del Estado o del Gobierno, Pierre (2013) argumenta que hay una "construcción de raza» poscolonial: "Un espacio poscolonial moderno es siempre un espacio racializado; es un espacio donde las lógicas raciales y culturales continúan constituyéndose y reconstituyéndose en las imágenes, las instituciones y las relaciones del momento colonial estructurante»(2013: xii). De esta manera, Agbogbloshie es un espacio donde la colonización, la contaminación y la racialización están estrechamente entrelazadas por las prácti- 
cas emergentes y actuales de la poscolonialidad y la toxicidad, prácticas que son experimentadas por «cuerpos integrados» (Lock, 2015; Niewöhner, 2011).

Dar sentido a los residuos electrónicos o a otros «desechos que se gestionan» (Gregson y Crang, 2010: 1027) en la poscolonia requiere una comprensión más profunda de la dinámica del trabajo, el comercio y las formas duraderas de explotación e injusticia en Ghana. Como podría sugerir Pickren (2014), la construcción de un enfoque crítico de salud ambiental y corporalidad sobre el riesgo de los residuos electrónicos en Agbogbloshie debe «tener en cuenta las omisiones y/o ambigüedades de la ley, en particular en relación con las exenciones y exclusiones para ciertos materiales, así como el comercio y las condiciones laborales" (Pickren, 2014: 31). Además, los sectores económicos emergentes o que se están reorganizando en Ghana para ayudar al desarrollo "económico" - y el desguace de Agbogbloshie es un actor importante en el sector industrial urbano de Accra- se enfrentan a duros desafíos poscoloniales. En la poscolonia, como nos recuerda Mbembe, "casi ningún sector... está libre de corrupción y venalidad» (Mbembe, 2001: 85). En un Agbogbloshie poscolonial, los trabajadores aprenden y luchan para hacerse camino por la venalidad tóxica.

Un «sector» que ha emergido y se ha mantenido rápidamente en Ghana al menos desde 2005 es el sector del reciclaje de residuos electrónicos, un sector que ha llamado la atención de científicos de salud ambiental y ocupacional a nivel mundial. Las exposiciones humanas a los residuos electrónicos son muy complejas, con múltiples rutas y duraciones de exposición, pero también en relación con la actividad por la cual la persona está expuesta. Como han sugerido Grant, Goldizen y Sly (2013), las exposiciones a los residuos electrónicos se pueden clasificar en tres sectores: reciclaje informal, reciclaje formal y exposición a compuestos peligrosos desechados durante las prácticas de reciclaje. Los centros formales de reciclaje pueden ayudar a resolver este problema y proteger a los trabajadores de los posibles efectos en la salud de esta exposición a los residuos electrónicos. Sin embargo, son muy costosos de construir y poco comunes en muchos de los centros de residuos electrónicos del mundo. De hecho, Agbogbloshie, tal y como analizaré detalladamente más adelante, se ha convertido en un espacio experimental para el reciclaje "formalizado». En general, sin embargo, la falta de estas instalaciones de reciclaje formalizadas y «más verdes» pone en mayor riesgo a los residentes que viven a cierta distancia de los espacios informales de reciclaje, a través de los alimentos, el agua y el suelo contaminados por las actividades de reciclaje de residuos tóxicos. Como han encontrado varias investigaciones, las 
rutas de exposición más comunes en estas áreas son a través de la inhalación, la ingestión y el contacto dérmico (Grant, Goldizen y Sly, 2013; Little, 2016) $)^{2}$.

Para algunos, el mercado del reciclaje de productos electrónicos ha creado empleos y ha reducido los niveles de pobreza en algunas partes del mundo, pero los residuos que crea este mercado de «reciclaje» son peligrosos para los sistemas sociales y ambientales. Sabiendo esto, los países desarrollados han adoptado sistemas de gestión aptos para ayudar a desviar y sacar los residuos de los países productores de artículos electrónicos. Posteriormente, estos residuos se transportan a países en desarrollo como Ghana, que tiene un sector informal que puede asimilar estos residuos y ayudar a reciclarlos para obtener ganancias. Sin embargo, Ghana carece de la legislación y la infraestructura adecuadas para manejar adecuadamente estos flujos de eliminación y «donación» de residuos electrónicos (Fuhriman, 2008). Del cuarenta al sesenta por ciento de los residuos electrónicos se reciclan, y el 95\% de ellos se reciclan de manera informal o en un sector laboral donde las preocupaciones por el medio ambiente y la seguridad humana no son una prioridad o no están bajo ninguna dirección formal. Muchos ghaneses estarían de acuerdo en que reemplazar el sector informal por un sector formal no sería práctico porque la mayoría del país depende del trabajo informal.

Según Oteng-Ababioa y Amankwaab (2014: 189), "los investigadores describen la recolección y el tratamiento de residuos electrónicos como un sistema altamente complejo, en el que el flujo de materiales incluye una gran variedad de actores interconectados". La recolección de desechos se ha consolidado como parte de una economía urbana que proporciona empleo a muchas de las personas más marginadas de Ghana. La recolección de residuos electrónicos puede ser difícil y también puede ser caro asegurar que llegue a las instalaciones de tratamiento adecuadas. Es aquí donde los investigadores observan el mayor solapamiento entre el mercado formal e informal. Ghana recibe más de 215.000 toneladas de residuos del mundo desarrollado cada año y el sector del reciclaje informal domina la mayor parte del país sin ninguna regulación. Algunos sugieren que el modelo ideal para el reciclaje en Ghana es integrar los sectores infor-

2. En China, por ejemplo, donde los estudios sobre residuos electrónicos tienen un recorrido más largo, se han realizado 165 estudios que ilustran la correlación entre las exposiciones a los residuos electrónicos y la salud física y mental, y los impactos en el desarrollo neurológico. De estos 165 estudios, 23 presentaron asociaciones tanto en salud física como mental, mientras que 16 estudios ilustraron asociaciones sólidas entre la exposición a residuos electrónicos y la salud física, mostrando efectos en la función tiroidea, la salud reproductiva, la función y el crecimiento pulmonar, y cambios adversos en las funciones celulares (Grant, Goldizen y Sly, 2013). 
males y formales para combinar los intereses de todos los grupos que participan en la economía de los residuos electrónicos, una economía que es responsable de aproximadamente el $95 \%$ de los residuos reciclados en Ghana (Oteng-Ababioa y Amankwaab, 2014).

Descifrar la experiencia del trabajo tóxico en Agbogbloshie pone el foco sobre numerosas cuestiones críticas en el ámbito de la salud ambiental: ¿a qué hacen frente los cuerpos y las subjetividades en un contexto urbano poscolonial tóxico? ¿Cómo se relacionan los cuerpos y los entornos, y cómo se narra y se hace legible esa relación?

\section{Narrativas corporalizadas sobre residuos electrónicos}

Dar sentido a la corporalidad tóxica y la permeabilidad en Agbogbloshie requiere ir "más allá del cuerpo en sí» (Lock y Farquhar, 2007), una perspectiva antropológica sobre el cuerpo que fomenta la discusión acerca de «una carnalidad vivaz repleta de palabras, imágenes, sentidos, deseos y poderes» (Farquhar y Lock, 2007: 15). No nos limitamos a enfrentarnos a cuerpos biologizados o culturalizados, sino a cuerpos ubicados e «integrados» (Lock, 2015; Niewöhner, 2011). De esta manera, Agbogbloshie es un espacio urbano en el que los "devenires biosociales» (Ingold y Palsson, 2013) persisten en un entorno de extrema contaminación y múltiples formas de riesgo tóxico. Especialmente en nuestra era epigenética inundada químicamente, todas las etnografías de la toxicidad se enfrentan al desafío de lidiar con las "multiplicidades inextricables entre los cuerpos materiales y los entornos pasados y presentes: variables históricas/sociales/ politicas y subjetividades» (Lock, 2015: 153). Al explorar la influencia de la epigenética entre los toxicólogos de la reproducción en China, Lamoreaux (2016) hizo avanzar el debate antropológico sobre epigenética al plantear la pregunta: "¿Y si el medio ambiente es una persona?" (Lamoreaux, 2016; énfasis mío), lo que significaría que el individuo puede tanto ser como representar al ambiente tóxico. El límite entre cuerpo y medio ambiente, entre persona y entorno, en este caso, se disuelve, lo que lleva a una relectura crítica del individuo como entorno y como señal ambiental que renueva "la urgencia sobre las relaciones entre el entorno material del cuerpo y su constitución [epigenética] interna» (Landecker, 2016: 91). También pone en cuestión la creciente convergencia de las políticas bio, eco e incluso geo-ontológicas (Povinelli, 2016) de los ambientes tóxicos en general. La tierra, el cuerpo, la economía y las sustancias tóxicas están entrelazadas en centros de reciclaje electrónico como Agbogbloshie, una situación que requiere un enfoque empírico crítico de los límites cuerpo-entorno y la naturaleza permeable de estos límites. 
En un esfuerzo por transitar estos límites corporales porosos, voy a referirme a las formas en que los trabajadores de residuos electrónicos mencionan no solo los estados de toxicidad «internos» (por ejemplo, dolores de pulmón y corazón), sino también las dolencias «externas» más visibles (por ejemplo, problemas dérmicos) para dar sentido y hacer legible su propia corporalidad tóxica. Esto implica preguntar cómo llegamos a conocer la experiencia corporal de la toxicidad y cuáles son, de hecho, los límites observables entre los cuerpos y los entornos, entre el objeto y el sujeto, en Agbogbloshie. También supone preguntarnos cómo o hasta qué punto la etnografía revela estos límites políticos al centrarse en la experiencia vivida y la identidad de quienes trabajan y viven en la primera línea de la toxicidad.

Para recorrer estas cuestiones importantes, quiero basarme en algunos hallazgos de la investigación etnográfica realizada en Agbogbloshie en los veranos de 2015 y 2016. Compuesta por cuerpos integrados y subjetividades vividas que lidian con un paisaje humeante, ardiente y peligroso de recuperación extrema de chatarra, el centro de atención de mi experiencia etnográfica en Agbogbloshie han sido las relaciones de poder en la intersección de cuerpos, toxinas, economías y estrategias de intervención «verde». Mi proyecto rastrea la experiencia vivida de un pequeño grupo de trabajadores que extraen cobre de productos electrónicos desechados, pero para los fines de este artículo me centraré en un trabajador en particular. En mi primera visita al campo, en julio de 2015, conocí a Ibrahim, un joven trabajador de Savelugu, que se encuentra en la región de Dagbon, en el norte de Ghana. Tenía veinte años cuando nos conocimos, y cuando regresé para el trabajo de campo en 2016 estaba casado y tenía un bebé de una semana de edad. Ibrahim pertenece a la tribu Dagomba, al igual que muchos de sus compañeros de trabajo en Agbogbloshie, y su familia vive en Old Fadama. De la misma forma que otros jóvenes de Savelugu, él viaja a Agbogbloshie durante tres meses seguidos, dos o tres veces al año. Más allá de las dificultades de ganarse la vida como joven trabajador migrante y de ser padre primerizo con poca educación formal, el «sufrimiento ambiental» (Auyero y Swistun, 2009) y la aflicción son factores estresantes adicionales.

Ibrahim me ha explicado varias veces durante mis visitas que su salud no es buena. Menciona su lucha con lo que él llama "tos de pecho», y también tiene dificultades para dormir. Me dice que pelea contra dolores de cabeza crónicos y agotamiento de cuerpo entero. Epidemiólogos del extranjero y de los Servicios de Salud de Ghana, la principal agencia de salud pública del país, han recogido su sangre y orina para estudios epidemiológicos. Como se señaló anteriormente, estos estudios se han publi- 
cado en revistas científicas creíbles, pero Ibrahim no tiene idea de cuáles son los resultados, ni qué están haciendo los investigadores con su sangre y orina, ni el porqué de la recolección rutinaria (anual) de esta materia corporal entre los trabajadores de residuos electrónicos. No tiene los datos de laboratorio sobre los niveles de plomo y cadmio en su cuerpo, pero sabe que el trabajo con residuos electrónicos lacera y quema su piel. Cuando Ibrahim y yo nos conocimos por primera vez en 2015 y le pregunté acerca de su salud, señaló sus quemaduras y cicatrices para mostrarme los impactos ambientales de su trabajo en la salud. Para él, los problemas de salud revelan todo lo que uno necesita saber sobre la dura realidad de trabajar en Agbogbloshie. Me lo expuso claramente durante una entrevista en 2016, poco después de que naciera su primer hijo: «Mire esto. Esto ser eso. Los residuos electrónicos. Se quema mi cuerpo. Aquí quemamos nuestros cuerpos. Nos duele. Sabemos que no es bueno. Pero, ¿cómo vivimos? La vida en el norte es dura. Necesitamos esto aquí. Mi cuerpo es malo. Mal cuerpo haciendo este trabajo». Para Ibrahim, vivir en uno de los «lugares más contaminados de la Tierra» se considera la mejor de dos opciones: esforzarse en el norte con un mercado agrícola débil y no ganar dinero, o resistir en el desguace de Agbogbloshie con exposiciones tóxicas extremas y ganar algo de dinero. Hacer frente a la toxicidad del trabajo con residuos es una cuestión de supervivencia económica, tal vez incluso una cuestión de vitalidad y resiliencia bioeconómica. Pero el desguace es un lugar donde no solo florece la economía informal, sino donde también se mantienen «medios de vida distribuidos» (Ferguson, 2015). En este sentido, la situación del trabajo asalariado lowcost de Agbogbloshie no es simplemente una situación económica, porque detrás de la práctica económica hay una lógica social de distribución más profunda: "la distribución es una actividad social crucial que es constitutiva del orden social (y no solo del económico). En consecuencia, debemos prestar atención a la idea de la distribución como una actividad social necesaria y valiosa» (Ferguson, 2015: 90). Cuando Ibrahim me habla de ganar dinero y volver a su aldea en el norte, está describiendo su plan de vida distributivo. Él está explicando su «economía humana» (Hart, Laville y Cattani, 2010) y cómo su trabajo productivo es más que solo suyo. En otras palabras, "ser alguien [sigue] implicando pertenecer a alguien» (Ferguson, 2015: 148).

Además de distribuir su salario a su familia, Ibrahim también comparte fotos de sus lesiones conmigo como forma de estar en contacto, pero también para mostrar los riesgos para la salud ambiental, que hablan directamente de la política vital de la permeabilidad y los riesgos dérmicos de la quema de cobre (ver Figura 3). 


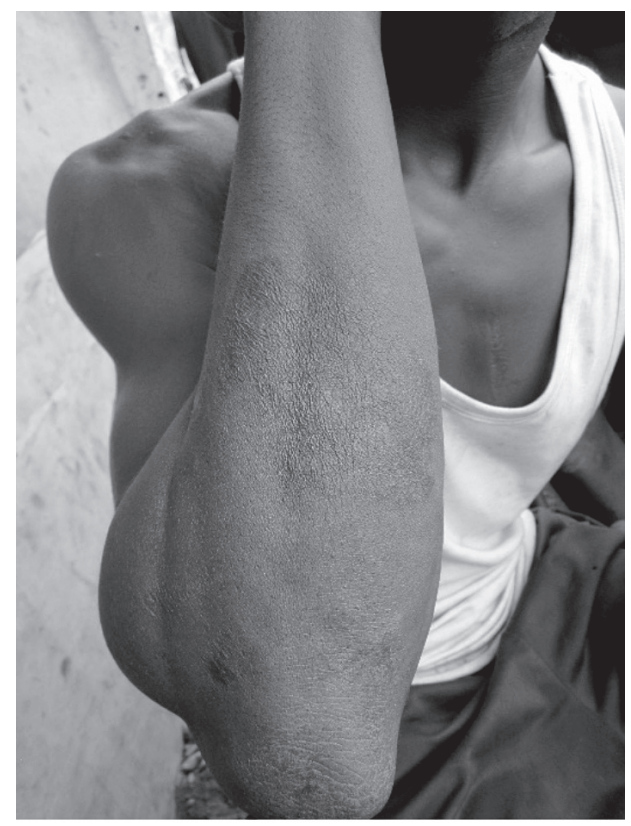

Figura 3. Cicatriz de Ibrahim de la quema de cables de cobre. Fotografía del autor.

Rodeado de metales tóxicos y llamaradas, Ibrahim experimenta riesgos más allá de la bioacumulación diaria de metales pesados. Se enfrenta, ante todo, a los riesgos de los incendios y el humo, en general, y de la incineración abierta de los innumerables materiales a base de petróleo que abarrotan el desguace. Su cuerpo quemado es simbólico del «nuevo materialismo» de la antropología del cuerpo; un cuerpo que «es a la vez subjetivo y objetivo, carnal y consciente, observable y legible» (Farquhar y Lock, 2007: 11). El riesgo tóxico se hace legible a través del cuerpo, la piel, la superficie del cuerpo integrado.

Las narrativas sobre toxicidad y riesgo en Agbogbloshie existen junto a las intervenciones y discursos de salud ambiental de las ONG. En 2014, Agbogbloshie se convirtió en el lugar donde construir un centro de reciclaje de residuos electrónicos «modelo» para hacer que el reciclaje fuera más seguro y respetuoso con el medio ambiente. El objetivo principal: «eliminar la quema en Agbogbloshie». Con el apoyo de una serie de agencias gubernamentales y no gubernamentales, las nuevas instalaciones de reciclaje, recientemente nombradas Centro de Reciclaje Agbogbloshie, tienen un objetivo claro de reducción de ries- 
gos (Figura 4). La lista de agencias y organizaciones asociadas es larga: la Comisión Europea, la Organización de las Naciones Unidas para el Desarrollo Industrial, la Alianza Mundial para la Salud y la Contaminación, el Ministerio de Medio Ambiente, la Agencia de Protección Ambiental de Ghana, los Servicios de Salud de Ghana, la Autoridad Nacional de Juventud, PureEarth.org, Green Advocacy Ghana, y la Asociación de Comerciantes de Residuos de Accra. En resumen, la nueva instalación pretende reducir los riesgos para la salud de la quema de cables — solo una de las muchas fuentes de contaminación del aire en Agbogbloshie- mediante el uso de máquinas automáticas para pelar cables recubiertos de varios tamaños que contengan cobre y otros materiales valiosos, aunque tóxicos. Los residuos que se reciben son principalmente cables eléctricos grandes, con base de aluminio, tanto de la compañía eléctrica de Ghana como de países vecinos como Burkina Faso.

Los manojos de cables que recogen y queman los trabajadores tienen un diámetro mucho menor y provienen de pequeños electrodomésticos, automóviles, autobuses y camiones desechados: cualquier residuo electrónico urbano que contenga cobre. Estos paquetes de cables varían en tamaño y tienen un valor de entre 8 y 10 cedi de Ghana (aproximadamente entre 2 y 2,5 dólares), aunque los valores del mercado de metales tienen un impacto directo en los precios locales del cobre. Los trabajadores tardan entre 10 y 15 minutos en quemar todo el aislamiento de plástico. Luego, el cable de cobre se embolsa y se vende a los comerciantes de chatarra, generalmente nigerianos, que venden el cobre para su exportación en Tema, el puerto industrial de Accra, que se encuentra a unas 20 millas al este del centro de la ciudad. El cobre en bruto se envía luego al extranjero para satisfacer las demandas de desarrollo en China e India.

Como agentes marginados en esta sucesión de eventos de recuperación de metales y productos, Ibrahim y sus compañeros de trabajo son conscientes de la contaminación del aire que crean. Son conscientes porque todo es visible, observable, y está a la vista. Como explicó un trabajador: "Lo que hacemos aqui todos lo pueden ver. Puedes ver nuestro humo. Estamos a la vista. Otras industrias están más ocultas y también lo está su contaminación. La gente se queja del humo que se produce aquí. Nuestro trabajo lo ves. Aquí puedes ver cómo va». 


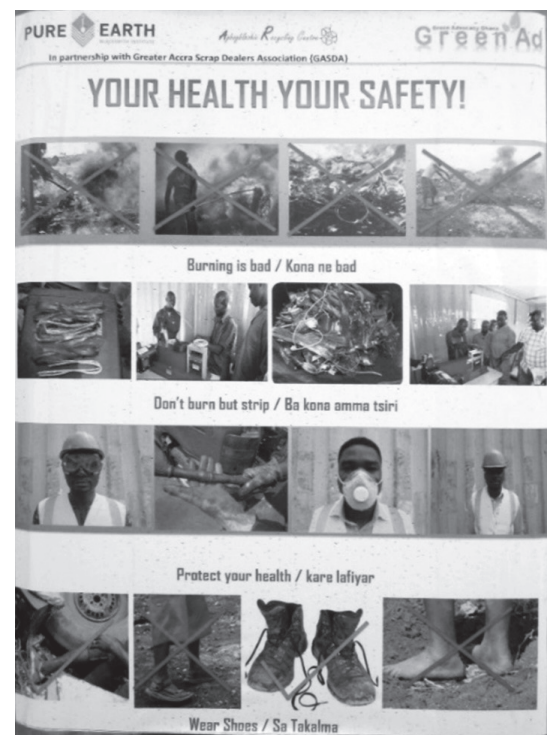

Figura 4. Póster de promoción de la salud en Agbogbloshie. Fotografía del autor.

Como se ve en la Figura 4, la campaña de promoción de la salud de Pure Earth se puede leer como un enfoque de «culpabilización de la víctima», donde a los trabajadores se les dice directamente que «la quema es mala» y que deberían "usar zapatos» y preocuparse por su salud. La campaña tiene como objetivo ofrecer soluciones fáciles de entender para las prácticas negativas del trabajo informal con residuos electrónicos. Si bien se podría decir más acerca de las dimensiones biopolíticas de este plan de promoción de la salud — particularmente, sobre las fuerzas combinadas de la autovigilancia y la formulación del reciclaje formal dentro de una narrativa de buenas prácticas de salud-, quiero señalar que, en realidad, el enfoque de Pure Earth para formalizar el reciclaje en Agbogbloshie margina aún más a los trabajadores que queman porque no tienen acceso directo a las instalaciones, no están formados para usar los granuladores para pelar cables, usar los granuladores hace más lento el proceso de extracción de cobre, usar la instalación cuesta dinero porque los granuladores funcionan con electricidad — un problema de infraestructura energética constante en Ghana-, y la instalación procesa principalmente cables de gran diámetro, provenientes de la empresa de servicios eléctricos de Ghana. El proyecto de Pure Earth opera bajo la idea «progresista» de que puede ser beneficioso tanto para los trabajadores como para el medio ambiente, pero muchos de los trabajadores que he 
entrevistado se muestran escépticos acerca de la eficacia y la sostenibilidad del proyecto. No importa cuánto se formalice el sector de reciclaje de residuos electrónicos, me dice un trabajador: "esta instalación no es buena para mí ni para muchos de nosotros. Será buena para alguien, pero todos aqui saben que el Gobierno quiere que nos vayamos. Desalojan a nuestra gente [en Old Fadama] que trabaja aqui. Pero nosotros hacemos el reciclaje de residuos electrónicos. Y ya ves, nadie se preocupa por nosotros aqui».

\section{Hacia una corporalidad tóxica poscolonial}

Agbogbloshie presenta algunas de las políticas de justicia ambiental más crudas y duras de nuestra época. Para los académicos de la «justicia en salud ambiental» (Masuada, Poland y Baxter, 2010; Miller y Wesley, 2016), no existe una separación lógica entre el cuerpo y las formas históricas y continuas de discriminación y violencia estructural que marcan el cuerpo. Como sugieren Miller y Wesley (2016: 75):

\footnotetext{
[El] enfoque de justicia en salud ambiental lleva a aquellos normalmente excluidos de la toma de decisiones ambientales al ámbito de la construcción de conocimientos científicamente rigurosos y socialmente relevantes para legitimar los esfuerzos comunitarios de justicia ambiental. Un enfoque de justicia en salud ambiental, por lo tanto, buscaría igualar el terreno de juego en términos de qué conocimientos "cuentan» en las decisiones de políticas que afectan a las comunidades marginadas.
}

Los cuerpos e identidades que logran ganarse la vida en Agbogbloshie se enfrentan a innumerables formas de estrés e injusticia social, económica y ambiental (Akese y Little, 2018). Por lo tanto, para comprender totalmente la política y la permeabilidad de los tóxicos en Agbogbloshie, me gustaría llamar la atención sobre el término corporalidad poscolonial tóxica para honrar el hecho de que Agbogbloshie es también un lugar y un espacio de micropolítica y poder poscolonial. Es un término flexible que se puede usar para fomentar los estudios de la relación cuerpo-tóxicos-medio ambiente en espacios y lugares poscoloniales contaminados.

En un retorno al estribillo original del colonialismo y la posterior etapa colonialista llamada "productiva", incluso en la era poscolonial, Mbembe (2001: 113) nos recuerda que "el colonialismo era, en gran medida, una forma de disciplinar los cuerpos con el objetivo de hacer un mejor uso de ellos, la docilidad y la productividad yendo de la mano". Esto es útil para pensar por qué Ibrahim y sus compañeros de trabajo 
migran de Savelugu a Accra para trabajar y «producir» en Agbogbloshie. Pueden ser más productivos y obtener mejores salarios trabajando en Agbogbloshie, ya que la venta de productos agrícolas en el norte de Ghana se considera una batalla económica perdida, especialmente para trabajadores como Ibrahim, que están recién casados, son padres primerizos y trabajan para tratar de mantener a sus familias. Él siente que su trabajo en Savelugu es inútil, a pesar de que su capital social en su pueblo es alabado porque su padre es un subjefe e Ibrahim mismo es tamborilero en el palacio del jefe de Savelugu, el Yoo Naa. Él ve el valor de migrar a Agbogbloshie porque es allí donde puede ganar dinero, un dinero que lleva a su padre y a otros familiares de Savelugu varias veces al año.

Si bien se pueden hacer infinidad de críticas a las ONG, especialmente por su participación en el África neoliberal a gran escala (Ferguson, 2006), Agbogbloshie presenta un caso único en el que los propios cuerpos son vistos como el objetivo ideal de la intervención en salud ambiental. A pesar de la importancia de los esfuerzos de mitigación de la contaminación, mi investigación etnográfica muestra que la preocupación más apremiante de los trabajadores es su capacidad para trabajar y tener un medio de vida económico que pueda mantener a sus familias. Estos trabajadores saben que sus cuerpos están en primera línea del frente tóxico, sopesan sus opciones lo mejor que pueden, pero muchos sienten que no importa cuánto se esfuercen, no pueden escapar de la pobreza y la marginación a la que se enfrentan en los tiempos poscoloniales de Ghana. El uso de la investigación etnográfica para comprender mejor las diversas políticas de vida social, ambiental y económica de los residuos electrónicos en el África poscolonial pone de manifiesto diversas políticas poscoloniales basadas en complejas narrativas sobre sufrimiento económico y ambiental. En Agbogbloshie en particular, la etnografía del trabajo y la toxicidad de los residuos electrónicos se convierte en una cuestión de transitar por «necropoliticas» industriales de alta tecnología (Mbembe, 2003), una cuestión de recicladores de residuos electrónicos realmente vivos, comprometidos y no obsoletos que trabajan con «materia vibrante» (Bennett, 2010) en la intersección y la frontera de la poscolonización y la contaminación. Mientras se enfrentan a las cargas corporales de la poscolonialidad tóxica, estos trabajadores de la basura electrónica son también sujetos y objetos de extrema precariedad económica y ambiental que desean una vida mejor en la poscolonia. Es en este sentido que este artículo busca llevar la conversación sobre políticas de salud ambiental en Agbogbloshie hacia el punto de vista de la corporalidad poscolonial tóxica. Como concepto crítico de salud ambiental que tiene como objetivo contribuir a los análisis sobre las relaciones cuerpo-medio ambiente en un contexto de margi- 
nalidad poscolonial, también puede emplearse para dar forma al análisis antropológico de la permeabilidad, en general, y de los límites permeables entre los residuos electrónicos, la toxicidad y el trabajo poscolonial en particular. De la misma manera que otros trabajos de antropología de los residuos exigen ir más allá del riesgo y los residuos mismos para avanzar en las teorías sociales (ver Reno, 2011 y 2014), este término también puede poner la atención sobre la necesidad de ir "más allá» de las narrativas dominantes sobre la toxicidad que moldean las percepciones e imágenes de Agbogbloshie y, en cambio, promover ideas sobre este dinámico mercado que explican más directamente cuál es el lugar de las narraciones y los cuerpos en la conformación de las teorías de las relaciones y fronteras entre el cuerpo y el entorno.

\section{Referencias}

Akese, G.A. y Little, P.C. (2018). Electronic Waste and the Environmental Justice Challenge in Agbogbloshie. Environmental Justice, 11(2): 77-83.

Alter, J.S. (2016). Medicine, Alternative Medicine, and Political Ecologies of the Body. En A Companion to the Anthropology of Environmental Health. M. Singer, Ed. Malden, MA: Wiley and Sons.

Asante, K.A.; Adu-Kumi, S. y Nakahiro, K. (2011). Human exposure to PCBs, PBDEs and HBCDs in Ghana: Temporal variation, sources of exposure and estimation of daily intakes by infants. Environment International, 37: 921-928.

Asante, K.A.; Agusa, T. y Biney, C.A. (2012). Multi-trace element levels and arsenic speciation in urine of e-waste workers from Agbogbloshie, Accra in Ghana. Science of the Total Environment, 424: 63-73.

Auyero, J. y Swistun, D.A. (2009). Flammable: Environmental Suffering in an Arentine Shantytown. Oxford: Oxford University Press.

Bennett, J. (2010). Vibrant Matter: A Political Ecology of Things. Durham: Duke University Press.

Caravanos, J., Clarke, E.; Fuller, R. y Lambertson, C. (2011). Assessing Worker and Environmental Chemical Exposure Risks at an e-Waste Recycling and Disposal Site in Accra, Ghana. Journal of Health and Pollution, 1(1): 16-25.

Caravanos, J.; Clarke, E. y Osei, C. (2013). Exploratory Health Assessment of Chemical Exposures at E-Waste Recycling and Scrapyard Facility in Ghana. Journal of Health and Pollution, 3(4): 11-22.

Chen, A.; Dietrich, K.N. y Huo, X. (2011). Developmental Neurotoxicants in E-Waste: An Emerging Health Concern. Environmental Health Perspectives, 119(4): 431-438.

Daum, K.; Stoler, J. y Grant, R.J. (2017). Toward a More Sustainable Trajectory for E-Waste Policy: A Review of a Decade of E-Waste Research in Accra, Ghana. International Journal of Environmental Research and Public Health, 14(135): 1-18.

Fanon, F. (1963). The Wretched of the Earth. Atlanta: Grove Press. 
Farquhar, J. y Lock, M. (2007). Introduction. En Beyond the Body Proper: Reading the Anthropology of Material Life. M. Lock y J. Farquhar, Eds. Durham: Duke University Press.

Feldt, T.; Fobil, J.N. y Wittseipe, J. (2014). High levels of PAH-metabolites in urine of e-waste recycling workers from Agbogbloshie, Ghana. Science of the Total Environment, 466467: 369-376.

Ferguson, J. (2006). Global Shadows: Africa in the Neoliberal World Order. Durham: Duke University Press.

Ferguson, J. (2015). Give A Man a Fish: Reflections on the New Politics of Distribution. Durham: Duke University Press.

Fuhriman, D.N. (2008). Dangerous Donations: Discarded Electronics in Accra, Ghana. Tesis no publicada. Department of Geography, Penn State University.

Grant, R. y Oteng-Ababio, M. (2016). The global transformation of materials and the emergence of informal "Urban Mining" in Accra, Ghana. Africa Today, 62: 2-20.

Grant, K., Goldizen, F. y Sly, P. (2013). Health Consequences of Exposure to E-Waste: A Systematic Review. The Lancet Global Health, 1(6): 350-361.

Gregson, N. y Crang, M. (2010). Materiality and Waste: Inorganic Vitality in a Networked World. Environment and Planning A, 42(5): 1026-1032.

Hart, K.; Laville, J.L. y Cattani, A.D. (Eds.) (2010). The Human Economy: A Citizen's Guide. London: Polity Press.

Ingold, T. y Palsson, G. (Eds.) (2013). Biosocial Becomings: Integrating Social and Biological Anthropology. Cambridge: Cambridge University Press.

Lamoreaux, J. (2016). What if the environment is a person? Lineages of epigenetic science in a toxic China. Cultural Anthropology 31(2): 188-214.

Landecker, H. (2016). The social as signal in the body of chromatin. En Biosocial Matters: Rethinking Sociology-Biology Relations in the Twenty-First Century. M. Meloni, S. Williams y P. Martin, Eds. Oxford: Wiley Blackwell.

Lundgren, K. (2012). The global impact of e-waste: Addressing the challenge. International Labour Office, Programme on Safety and Health at Work and the Environment (SafeWork), Sectoral Activities Department (SECTOR). Geneva: International Labour Organization.

Little, P.C. (2016). On Electronic Pyropolitics and Pure Earth Friction in Agbogbloshie. En https://toxicnews.org/2016/11/08/on-electronic-pyropolitics-and-pure-earth-fric tion-in-agbogbloshie/. Accedido el 8 de marzo de 2017.

Liu, J., Xu, X. y Wu, K. (2011). Association between lead exposure from electronic waste recycling and child temperament alterations. Neurotoxicology, 32: 458-464.

Lock, M. y Farquhar, J. (Eds.) (2007). Beyond the Body Proper: Reading the Anthropology of Material Life. Duke: Duke University Press.

Lock, M. (2015). Comprehending the body in the era of the epigenome. Current Anthropology, 56(2): 151-177.

Masuada, J.R., Poland, B. y Baxter, J. (2010). Reaching for Environmental Health Justice: Canadian Experiences for a Comprehensive Research, Policy and Advocacy Agenda in Health Promotion. Health Promotion International, 25: 453-463. 
Mbembe, A. (2001). On the Postcolony. Berkeley: University of California Press.

Miller, D.S. y Wesley, N. (2016). Toxic Disasters, Biopolitics, and Corrosive Communities: Guiding Principles in the Quest for Healing in Flint, Michigan. Environmental Justice, 9(3): 69-75.

Niewöhner, J. (2011). Epigenetics: embedded bodies and the molecularisation of biography and milieu. BioSocieties, 6(3): 279-298.

Nkrumah, K. (1965). Neo-Colonialism: The Last Stage of Imperialism. New York: International Publishers.

Oteng-Ababioa, M. y Amankwaab, E.F. (2014). The e-waste conundrum: Balancing evidence from the North and on-the-ground developing countries' realities for improved management. African Review of Economics and Finance, 6(1): 181-204.

Peluso, N.L. y Watts, M. (2001). Violent Environments. Ithaca: Cornell University Press.

Povinelli, E.A. (2016). Geontologies. Durham, NC: Duke University Press.

Pickren, G. (2014). Political Ecologies of Electronic Waste: Uncertainty and Legitimacy in the Governance of E-Waste Geographies. Environment and Planning A, 46: 26-45.

Pierre, J. (2013). The Predicament of Blackness: Postcolonial Ghana and the Politics of Race. Chicago: University of Chicago Press.

Reno, J. (2011). Beyond Risk: Emplacement and the Production of Environmental Evidence. American Ethnologist, 38(3): 516-530.

Reno, J. (2014). Toward a New Theory of Waste: from 'Matter out of Place' to Signs of Life. Theory, Culture \& Society, 31(6): 3-27.

Sepúlveda, A.; Schluep, M. y Renaud, F.G. (2010). A review of the environmental fate and effects of hazardous substances released from electrical and electronic equipments during recycling: Examples from China and India. Environmental Impact Assessment Review, 30(1): 28-41.

Wittsiepe, J.; Fobil, J.N. y Till, H. (2015). Levels of polychlorinated dibenzo-p-dioxins, dibenzofurans (PCDD/Fs) and biphenyls (PCBs) in blood of informal e-waste recycling workers from Agbogbloshie, Ghana, and controls. Environment International, 79: 65-73. 